\title{
Pancreatic and Hepatic Metastasis of an Undiagnosed Choriocarcinoma: An Exceptional Cause of Haemoperitoneum in Young Women - Report of a Rare Case
}

\author{
Nishat Fatema ${ }^{a} \quad$ Neeru Vinod Arora ${ }^{a} \quad$ Fatma Majid Al Abri ${ }^{a}$ \\ Yaseer Muhammad Tareq Khan ${ }^{\mathrm{b}}$ \\ ${ }^{a}$ Department of Obstetrics and Gynaecology, Ibri Regional Hospital, Ibri, Sultanate of \\ Oman; ${ }^{b}$ Department of Medicine, Ibri Regional Hospital, Ibri, Sultanate of Oman
}

\section{Keywords}

Haemoperitoneum · Choriocarcinoma $\cdot$ Metastasis

\begin{abstract}
Haemoperitoneum in women of reproductive age usually suggests either ruptured ectopic pregnancy or ruptured ovarian cysts. Metastatic choriocarcinoma is considered the least common cause of haemoperitoneum in women of childbearing age. We report a rare case of pancreatic and hepatic metastasis of choriocarcinoma in a young, 30-year-old female who had delivered 10 months prior at term gestation with no previous history of gestational trophoblastic disease or molar pregnancy. She had a short history of fever and pain in the right hypochondrium, with findings of hypovolaemic shock due to intraperitoneal haemorrhage. Unfortunately, the patient expired with massive uncontrolled bleeding from liver metastasis despite 2 emergency laparotomies within $12 \mathrm{~h}$. This case report is an apt reminder to clinicians to include metastatic choriocarcinoma on the list of differential diagnoses for haemoperitoneum with a positive pregnancy test in women of reproductive age to diagnose early and to avoid life-threatening consequences.


 Oncology}

\section{Background}

Choriocarcinoma is a highly vascular and metastatic malignant gestational trophoblastic neoplasm usually metastasized through the haematogenous route. The incidence of gestational choriocarcinoma is approximately 1 in 20,000-40,000 pregnancies; approximately $50 \%$ of the cases occur following term pregnancy, $25 \%$ after hydatidiform mole, and the remaining $25 \%$ related to other gestational conditions [1].

Metastasis spreads commonly to the lung (80\%), vagina (30\%), and pelvis $(20 \%)$, and less commonly to the liver and brain $(10 \%$ each). Pancreatic metastasis of choriocarcinoma is rarely encountered in the literature, with very few reported cases of secondary pancreatic metastasis of choriocarcinoma [2].

Patients with a history of gestational trophoblastic disease (GTD) or molar pregnancy are usually kept under strict regular follow-up; however, the prognosis may be unfavourable for those who present with an acute condition, disseminated metastasis, haemorrhagic complications, and a delayed diagnosis.

We share our experience of pancreatic and hepatic metastasis of an undiagnosed case of choriocarcinoma that presented with a short history of vomiting and pain in the right hypochondrium, with findings of hypovolaemic shock due to intraperitoneal haemorrhage. Unfortunately, we lost the patient despite 2 emergency laparotomies for intractable profuse intraperitoneal haemorrhage due to ruptured hepatic metastasis of the choriocarcinoma.

\section{Case}

A young, 30-year-old woman (para 1) was escorted from a health centre to our obstetric triage unit with acute onset of lower abdominal pain and hypovolaemic shock preceded by 3 days of fever and right hypochondriac pain. There was no history of vaginal bleeding.

On arrival, she was conscious but haemodynamically unstable. She looked pale with cold and clammy skin; her pulse was 134 beats/min and blood pressure was 90/30 $\mathrm{mm} \mathrm{Hg}$. She had a history of an uncomplicated term spontaneous vaginal delivery 10 months earlier in our hospital. After delivery, she had had regular periods at 2-week intervals with no excessive bleeding and was not using any contraceptive methods. A physical examination revealed a mildly distended abdomen with generalized tenderness.

We immediately initiated a diagnostic workup and performed resuscitation with crystalloids and colloidal solution. The laboratory investigations revealed the following: blood group 0 Rh positive; positive urine pregnancy test; $\beta$-human chorionic gonadotropin (HCG) $49,200 \mathrm{mIU} / \mathrm{ml}$; haemoglobin $5.95 \mathrm{~g} / \mathrm{dl}$; and platelet count $125 \times 10^{9} / \mathrm{l}$. However, she had a deranged coagulation profile and liver function tests: the serum bilirubin level was 12.5 $\mu \mathrm{mol} / \mathrm{l}$, ALT $194 \mathrm{U} / \mathrm{l}$, and ALP $190 \mathrm{U} / \mathrm{l}$.

Bedside transabdominal ultrasound revealed a normal uterus with no intrauterine gestation. The ovaries appeared normal with no adnexal mass, but there was a significant quantity of free fluid in the pouch of Douglas and Morrison's pouch.

The patient's presentation and clinical signs were strongly suggestive of ruptured ectopic pregnancy warranting urgent exploratory laparotomy. On opening the abdominal cavity with a Pfannenstiel incision, more than 1 litre of blood with clots was aspirated from the peritoneal cavity. On examination, the uterus and ovaries were normal; however, the right fallopian tube was mildly congested and inflamed but not ruptured. 
We noted profuse fresh bleeding pouring from the left hypochondriac region and requested a general surgery consultation to rule out a surgical cause. The surgeon explored the abdominal viscera by extending the incision and removed another litre of blood clots from the left hypochondriac area. After removing the blood clots, multiple variably sized nodular foci were noted on both lobes of the liver, the largest one measuring around $4 \times 5 \mathrm{~cm}$ (fig. 1). Continuous active bleeding was observed from the ruptured nodules. Tissue for histopathology was carefully captured from a fragile nodule. On further exploration of the abdominal cavity, the bowels were found normal, but the body of the pancreas had nodular foci similar to those of the liver, measuring approximately $2 \times 1.5 \mathrm{~cm}$ (fig. 2). Haemostasis was secured after suturing the ruptured nodules of the liver, and empirical right-sided salpingectomy was carried out. Intraoperatively, the patient lost around 3 litres of blood, which was replaced by 6 units of whole blood and 7 units of fresh frozen plasma.

The primary operative diagnosis suggested pancreatic and hepatic haemangiomas. The patient was transferred to the intensive care unit after surgery; however, $12 \mathrm{~h}$ following the laparotomy, she again had severe intra-abdominal haemorrhage, and consequently a second exploratory laparotomy was performed to control the haemorrhage. Haemostasis could not be completely achieved, and therefore it was decided to transfer the patient to a tertiary care hospital for hepatic artery embolization after haemodynamic stabilization. She stayed in the intensive care unit under ventilator support, and since she remained critical, she could not be transferred to tertiary care. Despite all resuscitative measures, the patient expired $11 \mathrm{~h}$ after the second exploratory laparotomy due to haemorrhagic shock.

Histopathology of the liver confirmed neoplastic cells compatible with choriocarcinoma, but the fallopian tube was normal with no evidence of chorionic villi. A pancreatic tissue biopsy was not taken during surgery due to the high clinical suspicion of haemangioma. Autopsy to exclude other organ metastases could not be carried out for social reasons.

\section{Discussion}

Hepatic metastasis of choriocarcinoma occurs in only $10 \%$ of GTD cases, and pancreatic metastasis is extremely rare. Alvarez et al. [2] report a case of pancreatic and hepatic metastasis that was saved by pancreatic resection and chemotherapy. In 1989, Erb and Gibler [3] reported a similar case of ruptured hepatic metastasis of choriocarcinoma, who survived after hepatic lobectomy and chemotherapy.

However, Kang et al. [4] described 2 patients with hepatic metastasis of choriocarcinoma, one of whom died 17 days after admission despite hepatic artery embolization. The other patient, who had a history of hydatidiform mole 5 years earlier, also expired due to uncontrolled bleeding from ruptured hepatic metastasis.

Even though our patient had no history of GTD, it has to be stated that due to the highly vascular characteristics and haemorrhagic tendency of the primary or metastatic site of the tumour, surgical intervention is a concern, since a patient's condition may deteriorate rapidly and the patient may even collapse [3, 4].

Besides baseline laboratory investigations, imaging techniques such as ultrasound and computed tomography (CT) angiograms of the abdomen can help with the diagnosis. The typical CT angiographic findings of hepatic metastasis of choriocarcinoma include peripheral hepatic artery aneurysmal dilatation at the arterial phase and persistent vascular lakes at the venous phase, which can differentiate the condition from other hypervascular hepatic lesions [4]. 
One case of pancreatic metastasis was detected on a CT angiogram of the abdomen [2]. Once the diagnosis is confirmed by CT angiogram and serum $\beta$-HCG levels, chest X-ray and brain imaging should be performed to exclude lung and brain metastases [1].

In our case, the patient presented in an acute condition with hypovolaemic shock and haemoperitoneum; therefore we could not wait for further investigations to confirm the diagnosis. If a patient were haemodynamically stable and had a history of molar pregnancy or GTD, a diagnosis of choriocarcinoma could be predicted. According to the World Health Organization's clinical classification of GTD, patients whose $\beta$-HCG level exceeds 40,000 $\mathrm{mIU} / \mathrm{ml}$ and who have liver metastases are regarded as a high-risk group with poorprognosis metastatic GTD [1]. Our patient's $\beta$-HCG level was 49,200 $\mathrm{mIU} / \mathrm{ml}$, and she had disseminated hepatic metastasis.

A previous review reported that the overall 5-year survival rate with liver metastasis is less than $30 \%$, and another review reported that the most common cause of death in hepatic metastasis patients is haemorrhage $[5,6]$.

In GTD, the presence of hepatic metastasis is an adverse prognostic factor despite haemorrhagic complications. To date, transcatheter angiographic embolization of the hepatic arteries is the accepted treatment modality to control haemorrhage. The bleeding vessel can be selectively embolized using this technique, with immediate visualization of its effect, and it can be carried out under local anaesthesia and mild sedation. The use of embolization is advantageous in unstable patients, as it is less invasive and time-consuming than open surgery and can be repeated if necessary, with a lower chance of complications such as failure to stop bleeding, rebleeding, and postembolization syndrome. In stable patients, multichemotherapy can be started after embolization [7].

Unfortunately, we did not have an angiographic embolization facility at our institute at that time and failed to transfer the patient to a tertiary centre, as she was haemodynamically unstable. In women with a high-risk GTD, multidrug chemotherapy has been shown to be superior to single-drug chemotherapy [8].

On the basis of 17 cases of hepatic metastasis, Barber et al. [9] reported that the survival of patients with GTD increased from 17\% before 1986 to 55\% after 1986, when EMA/CO chemotherapy was introduced. EMA/CO chemotherapy includes etoposide, methotrexate, actinomycin D, cyclophosphamide, and vincristine with folinic acid rescue.

The prognosis of patients with GTD with liver metastasis is poor in all staging systems, though they do respond to chemotherapy $[7,10]$. Further research needs to be carried out to investigate whether a more definitive scoring system to identify groups of patients at high risk will help in disease management [10]. The recurrence rate is approximately $7-10 \%$ in high-risk GTD patients. Although $20 \%$ of these patients subsequently fail to respond to treatment and die, the rest may be cured by further chemotherapy. Recurrence commonly occurs within 1 year. The follow-up protocol used in the individual institute can be applied, as there are currently no specific guidelines on an appropriate schedule for follow-up. Monitoring is life-long for this group of patients, since it is not clear when it is safe to stop surveillance [5].

\section{Conclusion}

Although pancreatic and hepatic metastasis of choriocarcinoma is a rare cause of haemoperitoneum in women of childbearing age, clinicians should maintain vigilance about 
differential diagnoses of haemoperitoneum in this group of patients to avoid the potentially fatal outcome of haemorrhagic complications.

\section{Acknowledgement}

We would like to thank Dr. Basant Kumar Sahoo and Dr. Amr Fouad Mohd El Borgy (Department of Surgery, Ibri Regional Hospital) for their cooperation and for providing us with operative photographs.

\section{Statement of Ethics}

Ethics approval was provided by the Research and Studies Committee (DGHS), Al Dhahirah Governorate (Ref. No. MH/DGHS/DG/590/16).

\section{Disclosure Statement}

No conflicts of interest are declared.

\section{References}

1 Soper JT, Mutch DG, Schink JC: Diagnosis and treatment of gestational trophoblastic disease: ACOG Practice Bulletin No 53. Gynecol Oncol 2004;93:575-585.

2 Alvarez NR, Lambrou N, Solorzano CC: Metastatic choriocarcinoma to the pancreas. Am Surg 2005;71:330-332.

-3 Erb RE, Gibler WB: Massive hemoperitoneum following rupture of hepatic metastases from unsuspected choriocarcinoma. Am J Emerg Med 1989;7:196-198.

-4 Kang YJ, Oh JH, Yoon Y, Kim EJ, Kim DY, Kang HS: Hepatic metastasis from choriocarcinoma: angiographic findings in two cases. Korean J Radiol 2002;3:260-263.

5 Tse KY, Chan KKL, Tam KF, Ngan HYS: An update on gestational trophoblastic disease. Obstet Gynaecol Reprod Med 2012;22:7-15.

6 Lurain JR, Brewer JI, Mazur MT, Torok EE: Fatal gestational trophoblastic disease: an analysis of treatment failures. Am J Obstet Gynecol 1982;144:391-395.

-7 Lok CA, Reekers JA, Westermann AM, Van der Velden J: Embolization for hemorrhage of liver metastases from choriocarcinoma. Gynecol Oncol 2005;98:506-509.

-8 Wright JD, Mutch DG: Treatment of high-risk gestational trophoblastic tumors. Clin Obstet Gynecol 2003;46:593-606.

-9 Barber EL, Schink JC, Lurain JR: Hepatic metastasis in gestational trophoblastic neoplasia: patient characteristics, prognostic factors, and outcomes. J Reprod Med 2014;59:199-203.

10 Crawford RA, Newlands E, Rustin GJ, Holden L, A'Hern R, Bagshawe KD: Gestational trophoblastic disease with liver metastases: the Charing Cross experience. Br J Obstet Gynaecol 1997;104:105-109. 


\section{Case Reports in Oncology}

\begin{tabular}{l|l}
\hline Case Rep Oncol 2016;9:633-638 \\
\hline DOI: 10.1159/000449462 & $\begin{array}{l}\text { C 2016 The Author(s). Published by S. Karger AG, Basel } \\
\text { www.karger.com/cro }\end{array}$ \\
\hline
\end{tabular}

Fatema et al: Pancreatic and Hepatic Metastasis of an Undiagnosed Choriocarcinoma: An Exceptional Cause of Haemoperitoneum in Young Women - Report of a Rare Case

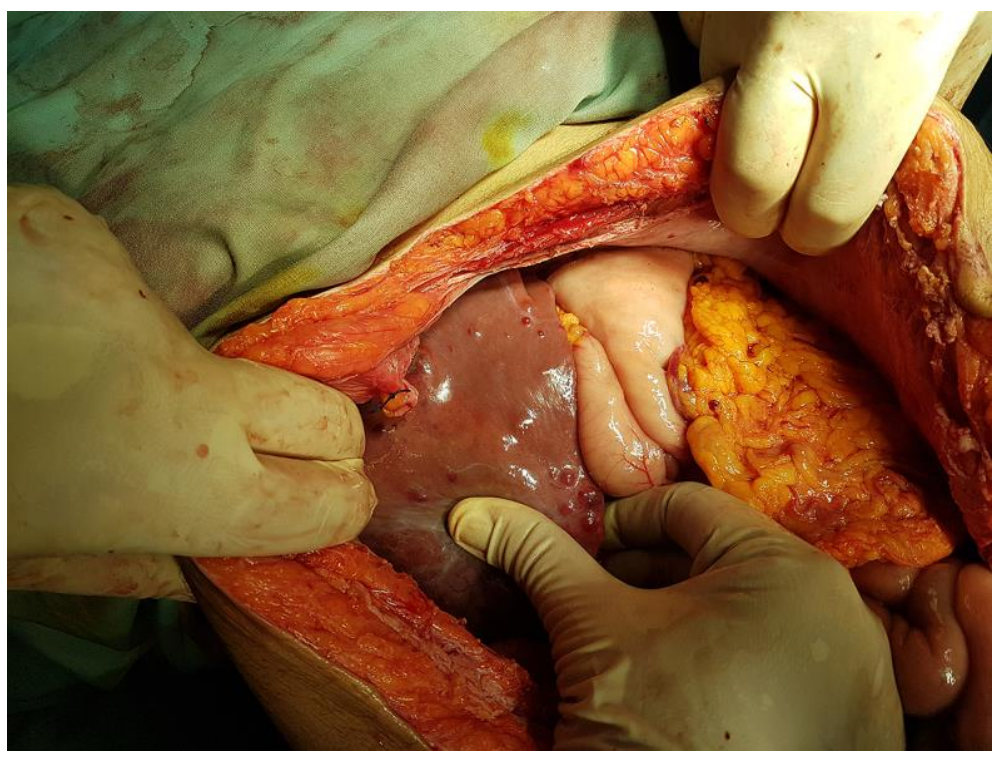

Fig. 1. Intraoperative finding: hepatic metastasis of choriocarcinoma.

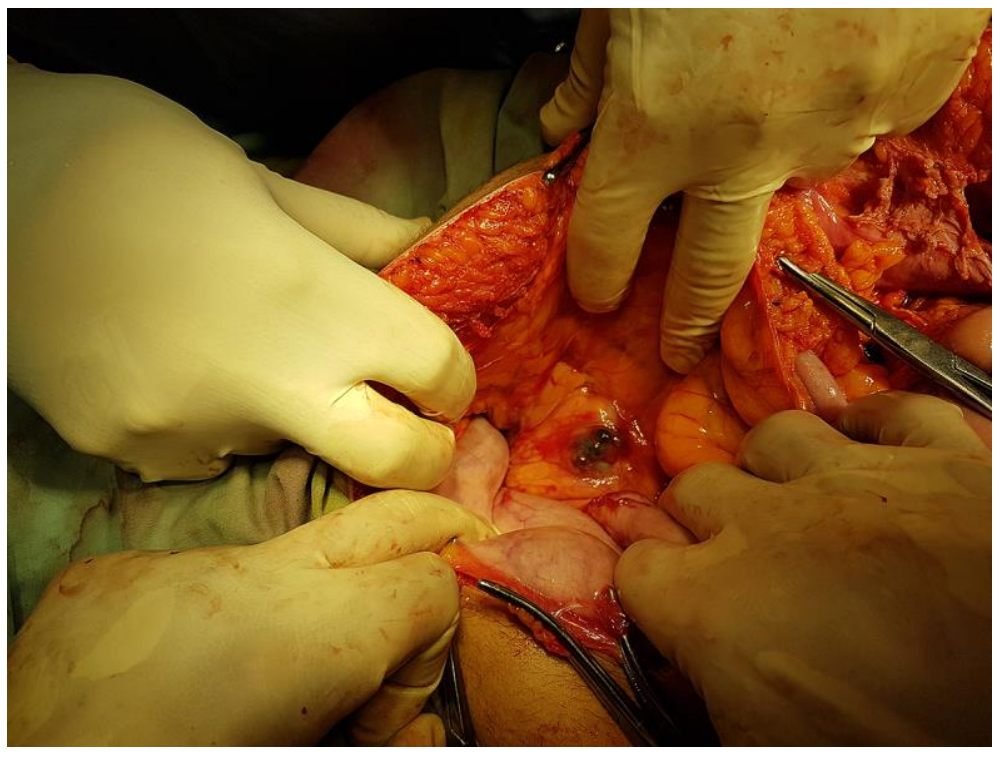

Fig. 2. Perioperative finding: pancreatic metastasis of choriocarcinoma. 